\title{
OBSTRUCTIONS TO THE EXISTENCE OF KÄHLER STRUCTURES ON COMPACT COMPLEX MANIFOLDS
}

\author{
IONUŢ CHIOSE
}

(Communicated by Lei $\mathrm{Ni}$ )

\begin{abstract}
We prove that a manifold in the Fujiki class $\mathcal{C}$ which supports a $i \partial \bar{\partial}$-closed metric is Kähler. This result implies that on a compact complex manifold in the Fujiki class $\mathcal{C}$ which is not Kähler there exists a nonzero $i \partial \bar{\partial}-$ exact, positive current of bidimension $(1,1)$.
\end{abstract}

\section{INTRODUCTION}

In HaLa, Harvey and Lawson proved that the obstruction to the existence of a Kähler metric on a given compact complex manifold $X$ of dimension $n$ is a positive, nonzero current of bidegree $(n-1, n-1)$ which is the $(n-1, n-1)$ component of a $d$-exact current on $X$. In general, such currents are not $d$-closed, therefore the theory of closed positive currents cannot be used to study them (although some results extending this theory to the case of $i \partial \bar{\partial}$-closed, positive currents do exist). The main result of this paper is that, in the case when $X$ is a manifold in the Fujiki class $\mathcal{C}$, the obstruction current can be chosen to be $d$-closed:

Theorem 0.1. Let $X$ be a compact complex manifold of dimension $n$ in the Fujiki class $\mathcal{C}$ and which is not Kähler. Then there exists a positive, nonzero current $T$ of

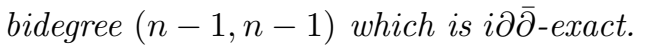

Theorem 0.1 follows immediately from

Theorem 0.2. Let $X$ be a compact complex manifold of dimension $n$ in the Fujiki class $\mathcal{C}$ and suppose there exists $\omega$ a strictly positive $(1,1)$ form on $X$ such that $i \partial \bar{\partial} \omega=0$. Then $X$ is a Kähler manifold.

The two theorems are generalizations to the analytic case of the algebraic case which was proved by Peternell $[\mathrm{Pe}$. Theorem 0.2 is similar to Moishezon's theorem which states that a Moishezon manifold which is Kähler is in fact projective.

A $(1,1)$ form $\omega$ as in Theorem 0.2 (i.e., positive defined, and $i \partial \bar{\partial}$-closed) is called a strong Kähler with torsion $(S K T)$ metric. See for instance [FiTo for an introduction to $S K T$ metrics. Therefore, Theorem 0.2 states that a manifold in Fujiki class $\mathcal{C}$ which supports an $S K T$ metric is in fact Kähler.

On surfaces, Theorems 0.1 and 0.2 are vacuous since any surface in the Fujiki class $\mathcal{C}$ is Kähler. But on 3 -folds, Theorem 0.1 implies that any closed obstruction contains a nonzero curve:

Received by the editors November 6, 2012.

2010 Mathematics Subject Classification. Primary 32J27; Secondary 32Q15.

The author was supported by a Marie Curie International Reintegration Grant within the $7^{\text {th }}$ European Community Framework Programme and the CNCS grant PN-II-ID-PCE-2011-3-0269. 
Theorem 0.3. Let $X$ be a compact complex 3 -fold in class $\mathcal{C}$ which is not Kähler

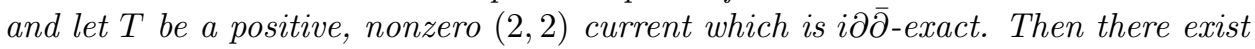
$C$ an irreducible curve in $X, \lambda>0$ and $R$ a closed positive $(2,2)$ current on $X$ such that $T=\lambda[C]+R$.

Theorem 0.1, combined with a result of Lamari [La, Théorème 3.2 (see Theorem 1.5 below) implies the following general existence theorem, which is a refined version of the Harvey and Lawson theorem:

Theorem 0.4. Let $X$ be a compact complex manifold of dimension $n$ such that:

(i) there is no nontrivial nef pluriharmonic current on $X$ of bidegree $(n-1$, $n-1)$, which is the $(n-1, n-1)$ component of a boundary,

(ii) there is no nontrivial positive, $\partial \bar{\partial}$-exact current of bidegree $(n-1, n-1)$ on $X$.

Then $X$ is Kähler.

Therefore, on a non-Kähler manifold, the obstruction is either nef (when the manifold is not in $\mathcal{C}$ ) or closed (when the manifold is in the Fujiki class $\mathcal{C}$ ).

For the proof of Theorem 0.2 , we first show that the cohomology class of the $i \partial \bar{\partial}$-closed form satisfies the numerical conditions of a Kähler class. The main result of DePă then implies that the cohomology class contains a Kähler current. We then proceed by induction on the dimension of the manifold to show that the cohomology class contains a Kähler form.

Theorem 0.1 follows at once from Theorem 0.2 by using a result of Harvey and Lawson HaLa].

\section{Preliminaries}

In this section we gather some results needed for the proof of the above results.

1.1. Intrinsic characterization of Kähler manifolds. The main result of HaLa] is the following:

Theorem 1.1. Let $X$ be a compact manifold of dimension $n$. Then $X$ is nonKähler iff there exists a nonzero positive current which is the $(n-1, n-1)$ component of a boundary.

In the same paper, the authors prove that the obstruction to the closedness of the obstruction current involves a strictly positive, $i \partial \bar{\partial}$-closed $(1,1)$ form:

Theorem 1.2. Suppose $X$ is a compact manifold of dimension $n$. Then $X$ admits a closed real $(1,1)$ form $\eta=\bar{\partial} \alpha+\omega+\partial \bar{\alpha}$, where $\omega$ is a strictly positive $(1,1)$ form and $\alpha$ is a $(1,0)$ form on $X$ iff $X$ does not support a nonzero, $d$-closed, positive current which is the $(n-1, n-1)$ component of a boundary.

1.2. Positive classes on compact manifolds. The main result of [DePă] is the following:

Theorem 1.3. Let $(X, \lambda)$ be a compact Kähler manifold and let $\{\eta\}$ be a $(1,1)$ cohomology class on $X$. Then $\{\eta\}$ is a Kähler cohomology class iff for every irreducible analytic set $Z \subset X, \operatorname{dim} Z=p$, and every $k=\overline{1, p}$,

$$
\int_{Z} \eta^{k} \wedge \lambda^{p-k}>0
$$


In order to construct a Kähler metric, we will need the following result from DePă:

Theorem 1.4. Let $X$ be a compact complex space and let $\{\eta\}$ be a cohomology class of type $(1,1)$ on $X$. Assume that $\{\eta\}$ contains a Kähler current $T$ and that the restriction $\{\eta\} \mid Y$ to every irreducible component $Y$ in the Lelong sublevel sets $E_{c}(T)$ is a Kähler cohomology class. Then $\{\eta\}$ is a Kähler cohomology class on $X$.

1.3. Manifolds in the Fujiki class $\mathcal{C}$. The manifolds in class $\mathcal{C}$ were first introduced by Fujiki as manifolds which are meromorphic images of Kähler manifolds $\mathrm{Fu}$ :

Definition 1.1. A compact complex manifold $X$ is in class $\mathcal{C}$ if there exists a complex Kähler space $Y$ and a surjective meromorphic map $h: Y \rightarrow X$.

There are several other ways of characterizing the manifolds in the Fujiki class. A current $T$ of bidegree $(n-1, n-1)$ on a compact complex manifold $X$ of dimension $n$ is nef pluriharmonic if it is a weak limit of Gauduchon metrics. A closed current of bidegree $(1,1)$ is a Kähler current if it dominates some strictly positive, smooth $(1,1)$ form. Then we have:

Theorem 1.5. Let $X$ be a compact complex manifold of dimension $n$. Then the following are equivalent:

(i) $X$ is in the Fujiki class $\mathcal{C}$,

(ii) there exists $Y$ a Kähler manifold and $h: Y \rightarrow X$ a proper transform of $X$ ( Va $)$,

(iii) there exists $T$ a Kähler current on $X$ ([DePă $]$,

(iv) if $R$ is an $(n-1, n-1)$ nef pluriharmonic current on $X$ which is the $(n-1, n-1)$ component of a boundary, then $R=0$ ([La $]$ ).

The Fujiki class $\mathcal{C}$ is stable under most natural operations, except under small deformations. The Hodge decomposition is valid on manifolds in the Fujiki class $\mathcal{C}$; in particular, the $\partial \bar{\partial}$ lemma is valid on such manifolds.

\section{Non-KäHLER MANifolds in the FuJiki Class $\mathcal{C}$}

In this section we prove Theorems 0.1 and 0.2 . We first need the following lemma, which will be used later to show that a certain cohomology class satisfies the numerical inequalities of a Kähler class:

Lemma 2.1. Let $X$ be a compact complex manifold of dimension $n, \eta=\partial \bar{\alpha}+\omega+\bar{\partial} \alpha$ be a closed $(1,1)$ form where $\alpha$ is a $(1,0)$ form on $X$ and $\omega$ is a strictly positive $(1,1)$ form on $X$, and $\lambda$ be a closed real $(n-k, n-k)$ form on $X$. Then

$$
\int_{X} \eta^{k} \wedge \lambda=\sum_{2 i+j=k}\left(\begin{array}{c}
k \\
j
\end{array}\right)\left(\begin{array}{c}
2 i \\
i
\end{array}\right) \int_{X} \omega^{j} \wedge(\partial \alpha \wedge \bar{\partial} \bar{\alpha})^{i} \wedge \lambda .
$$


Proof. Since $\eta$ is closed, it follows that $\partial \omega=\bar{\partial} \partial \alpha$ and $\bar{\partial} \omega=\partial \bar{\partial} \bar{\alpha}$. We prove the statement by induction on $k$. For $k=1$, the above equation becomes

$$
\int_{X} \eta \wedge \lambda=\int_{X} \omega \wedge \lambda
$$

and it follows from Stokes' theorem since $\lambda$ is closed. Suppose the formula is true for $k$. Then for $k+1$ we have

$$
\begin{gathered}
\int_{X} \eta^{k+1} \wedge \lambda=\int_{X} \eta^{k} \wedge \eta \wedge \lambda \\
=\sum_{2 i+j=k}\left(\begin{array}{c}
k \\
j
\end{array}\right)\left(\begin{array}{c}
2 i \\
i
\end{array}\right) \int_{X} \omega^{j} \wedge(\partial \alpha \wedge \bar{\partial} \bar{\alpha})^{i} \wedge(\bar{\partial} \alpha+\omega+\partial \bar{\alpha}) \wedge \lambda \\
=\sum_{2 i+j=k}\left(\begin{array}{c}
k \\
j
\end{array}\right)\left(\begin{array}{c}
2 i \\
i
\end{array}\right) \int_{X} \omega^{j+1} \wedge(\partial \alpha \wedge \bar{\partial} \bar{\alpha})^{i} \wedge \lambda \\
+\sum_{2 i+j=k}\left(\begin{array}{c}
k \\
j
\end{array}\right)\left(\begin{array}{c}
2 i \\
i
\end{array}\right) \int_{X} \bar{\partial} \alpha \wedge \omega^{j} \wedge(\partial \alpha \wedge \bar{\partial} \bar{\alpha})^{i} \wedge \lambda \\
+\sum_{2 i+j=k}\left(\begin{array}{c}
k \\
j
\end{array}\right)\left(\begin{array}{c}
2 i \\
i
\end{array}\right) \int_{X} \partial \bar{\alpha} \wedge \omega^{j} \wedge(\partial \alpha \wedge \bar{\partial} \bar{\alpha})^{i} \wedge \lambda .
\end{gathered}
$$

The third term in the above sum is the conjugate of the second term, so we focus only on the second term, call it $I_{2}$. Stokes' theorem implies that it is equal to

$$
\begin{aligned}
& I_{2}=\sum_{2 i+j=k} j\left(\begin{array}{c}
k \\
j
\end{array}\right)\left(\begin{array}{c}
2 i \\
i
\end{array}\right) \int_{X} \alpha \wedge \partial \bar{\partial} \bar{\alpha} \wedge \omega^{j-1} \wedge(\partial \alpha \wedge \bar{\partial} \bar{\alpha})^{i} \wedge \lambda \\
& +\sum_{2 i+j=k} i\left(\begin{array}{c}
k \\
j
\end{array}\right)\left(\begin{array}{c}
2 i \\
i
\end{array}\right) \int_{X} \alpha \wedge \partial \omega \wedge \omega^{j} \wedge \bar{\partial} \bar{\alpha} \wedge(\partial \alpha \wedge \bar{\partial} \bar{\alpha})^{i-1} \wedge \lambda,
\end{aligned}
$$

where we have used $\bar{\partial} \omega=\partial \bar{\partial} \alpha$ and $\bar{\partial} \partial \alpha=\partial \omega$. Now notice that

$$
\begin{gathered}
\partial\left[\sum_{2 i+j=k-1} \frac{j+1}{i+1}\left(\begin{array}{c}
k \\
j+1
\end{array}\right)\left(\begin{array}{c}
2 i \\
i
\end{array}\right) \bar{\partial} \bar{\alpha} \wedge \omega^{j} \wedge(\partial \alpha \wedge \bar{\partial} \bar{\alpha})^{i}\right] \\
\quad=\sum_{2 i+j=k} j\left(\begin{array}{c}
k \\
j
\end{array}\right)\left(\begin{array}{c}
2 i \\
i
\end{array}\right) \partial \bar{\partial} \bar{\alpha} \wedge \omega^{j-1} \wedge(\partial \alpha \wedge \bar{\partial} \bar{\alpha})^{i} \\
+\sum_{2 i+j=k} i\left(\begin{array}{c}
k \\
j
\end{array}\right)\left(\begin{array}{c}
2 i \\
i
\end{array}\right) \partial \omega \wedge \omega^{j} \wedge \bar{\partial} \bar{\alpha} \wedge(\partial \alpha \wedge \bar{\partial} \bar{\alpha})^{i-1}
\end{gathered}
$$

and again Stokes' theorem implies that the second term is equal to

$$
I_{2}=\sum_{2 i+j=k-1} \frac{j+1}{i+1}\left(\begin{array}{c}
k \\
j+1
\end{array}\right)\left(\begin{array}{c}
2 i \\
i
\end{array}\right) \int_{X} \omega^{j} \wedge(\partial \alpha \wedge \bar{\partial} \bar{\alpha})^{i+1} \wedge \lambda .
$$

Therefore

$$
\begin{gathered}
\int \eta^{k+1} \wedge \lambda=\sum_{2 i+j=k}\left(\begin{array}{c}
k \\
j
\end{array}\right)\left(\begin{array}{c}
2 i \\
i
\end{array}\right) \int_{X} \omega^{j+1} \wedge(\partial \alpha \wedge \bar{\partial} \bar{\alpha})^{i} \wedge \lambda \\
+2 \sum_{2 i+j=k-1} \frac{j+1}{i+1}\left(\begin{array}{c}
k \\
j+1
\end{array}\right)\left(\begin{array}{c}
2 i \\
i
\end{array}\right) \int_{X} \omega^{j} \wedge(\partial \alpha \wedge \bar{\partial} \bar{\alpha})^{i+1} \wedge \lambda .
\end{gathered}
$$


Now the formula for $k+1$ follows from rearranging the terms of the sums, along with the trivial identity

$$
\left(\begin{array}{c}
k+1 \\
j
\end{array}\right)\left(\begin{array}{c}
2 i \\
i
\end{array}\right)=\left(\begin{array}{c}
k \\
j-1
\end{array}\right)\left(\begin{array}{c}
2 i \\
i
\end{array}\right)+2 \frac{j+1}{i}\left(\begin{array}{c}
k \\
j+1
\end{array}\right)\left(\begin{array}{c}
2 i-2 \\
i-1
\end{array}\right)
$$

for $2 i+j=k+1$.

Now we can prove

Theorem 2.2. Let $X$ be a compact complex manifold of dimension $n$ in the Fujiki class $\mathcal{C}$ and suppose there exists $\omega$ a strictly positive $(1,1)$ form on $X$ such that $i \partial \bar{\partial} \omega=0$. Then $X$ is a Kähler manifold.

Proof. Note that since $X$ is in the Fujiki class $\mathcal{C}$, the $\partial \bar{\partial}$ lemma is valid on $X$, and therefore the condition $i \partial \bar{\partial} \omega=0$ implies the existence of $\alpha$ and $\eta$ as in Lemma 2.1 (apply the $i \partial \bar{\partial}$-lemma to the $d$-closed and $\partial$-exact form $\partial \omega$ ). We prove by induction on $n$ that $\{\eta\}$ is a Kähler cohomology class.

First assume that $X$ can be made Kähler by a single blow-up along a smooth submanifold $Y \subset X, \operatorname{dim} Y \leq n-2$. Denote by $\pi: \widetilde{X} \rightarrow X$ the blow-up of $X$ along $Y$, denote by $\widetilde{Y} \subset \widetilde{X}$ the exceptional divisor and suppose that $\widetilde{\lambda}$ is a Kähler form on $\widetilde{X}$. It is well-known that there exists a smooth $d$-closed $(1,1)$ form $\widetilde{u}$ on $\widetilde{X}$, in the cohomology class of $[\widetilde{Y}]$, such that $\widetilde{\omega}=\pi^{*} \omega-\varepsilon \widetilde{u}$ is strictly positive on $\widetilde{X}$ for some small $\varepsilon>0$. Moreover, $\widetilde{u}=[\tilde{Y}]+i \partial \bar{\partial} \widetilde{\psi}$, where $\widetilde{\psi}$ is smooth on $\widetilde{X} \backslash \tilde{Y}$. Set $\widetilde{\alpha}=\pi^{*} \alpha$ and $\widetilde{\eta}=\partial \overline{\widetilde{\alpha}}+\widetilde{\omega}+\bar{\partial} \widetilde{\alpha}$. Then we can use Lemma 2.1 for $\widetilde{\eta}$ on $\widetilde{X}$.

Note that

$$
(\partial \widetilde{\alpha} \wedge \bar{\partial} \overline{\widetilde{\alpha}})^{i}=(\partial \widetilde{\alpha})^{i} \wedge(\bar{\partial} \overline{\widetilde{\alpha}})^{i}
$$

is a weakly positive $(2 i, 2 i)$ form on $\tilde{X}$. Hence, when we multiply it with the strongly positive form $\widetilde{\omega}^{j} \wedge \widetilde{\lambda}^{k}$, we obtain a positive multiple of the volume form. Therefore we obtain that

$$
\int_{\widetilde{Z}} \widetilde{\eta}^{k} \wedge \widetilde{\lambda}^{p-k}>0
$$

for every irreducible analytic subset $\widetilde{Z} \subset \widetilde{X}, \operatorname{dim} \widetilde{Z}=p$ and every $k=\overline{1, p}$. Indeed, when $\widetilde{Z}$ is smooth, this follows from Lemma 2.1 .

In the case when $\widetilde{Z}$ is not smooth, we use Hironaka's resolution of singularities, and we obtain a manifold $\widetilde{X}^{\prime}$ which is a sequence of blow-ups with smooth centers of $\widetilde{X}$, and a smooth submanifold $\widetilde{Z}^{\prime}$ which resolves the singularities of $\widetilde{Z}$. It is clear that the integral in (2.2) is equal to the corresponding integral over $\widetilde{Z}^{\prime}$ and hence is nonnegative.

Theorem 1.3 implies that $\{\widetilde{\eta}\}$ is a Kähler cohomology class on $\widetilde{X}$; i.e., there exists $\widetilde{\varphi} \in \mathcal{C}^{\infty}(\widetilde{X}, \mathbb{R})$ such that $\widetilde{\eta}+i \partial \bar{\partial} \widetilde{\varphi}>0$.

Now we push everything forward to $X$. At the cohomology level, we have on $\widetilde{X}$ that

$$
\{\widetilde{\eta}\}=\pi^{*}\{\eta\}-\varepsilon\{[\widetilde{Y}]\} .
$$

The push-forward of $[\tilde{Y}]$ is 0 , so it follows that $\pi_{*}\{\widetilde{\eta}\}=\{\eta\}$ contains a Kähler current which is smooth on $X \backslash Y$. 
Note that a smooth submanifold of a manifold in Fujiki class $\mathcal{C}$ is also in Fujiki class $\mathcal{C}$. Hence, by induction, we obtain that the restriction of $\{\eta\}$ to $Y$ is a Kähler cohomology class, and by Theorem 1.4 it follows that $\{\eta\}$ is a Kähler cohomolgy class.

In general, suppose that $X$ can be made Kähler by a sequence of blow-ups with smooth centers $X_{r} \rightarrow X_{r-1} \rightarrow \ldots \rightarrow X_{1} \rightarrow X_{0}=X$, choose $r$ to be minimal, and suppose $r \geq 1$. We can easily construct a strictly positive $(1,1)$-form $\omega_{r-1}$ on $X_{r-1}$ such that $i \partial \bar{\partial} \omega_{r-1}=0$. Then $X_{r-1}$ is Kähler, contradicting the minimality of $r$.

On 3-folds we can prove a stronger result:

Theorem 2.3. Let $X$ be a 3-fold in the Fujiki class $\mathcal{C}$ and $\omega$ a strictly positive $(1,1)$ form on $X$ such that either $i \partial \bar{\partial} \omega \geq 0$ or $i \partial \bar{\partial} \omega \leq 0$. Then $X$ is Kähler.

Proof. Since $X$ is in the Fujiki class $\mathcal{C}$, there exists $T \geq \gamma$ a Kähler current on $X$, where $\gamma$ is a strictly positive $(1,1)$ form on $X$. If $i \partial \bar{\partial} \omega \geq 0$, then

$$
\langle i \partial \bar{\partial} \omega, \gamma\rangle \leq\langle i \partial \bar{\partial} \omega, T\rangle=\langle\omega, i \partial \bar{\partial} T\rangle=0 ;
$$

hence $i \partial \bar{\partial} \omega=0$ and similarly when $i \partial \bar{\partial} \omega \leq 0$. Therefore $i \partial \bar{\partial} \omega=0$ and the conclusion follows from Theorem 2.2 .

Now we can easily prove

Theorem 2.4. Let $X$ be a compact complex manifold of dimension $n$ in the Fujiki class $\mathcal{C}$ and which is not Kähler. Then there exists a positive, nonzero current $T$ of bidegree $(n-1, n-1)$, which is $i \partial \bar{\partial}$-exact.

Proof. By Theorem 1.2, it is enough to prove that if $X$ supports a strictly positive, $i \partial \bar{\partial}$-closed, $(1,1)$ form, then $X$ is Kähler. But this is just the statement of Theorem 2.2. The $i \partial \bar{\partial}$-exactness of $T$ follows immediately from the $\partial \bar{\partial}$ lemma.

\section{NON-KÄHLER 3-FOLDS}

In this section we show that on any 3 -fold in class $\mathcal{C}$ which is not Kähler there exists a curve which is part of the obstruction.

Theorem 3.1. Let $X$ be a 3-fold in class $\mathcal{C}$ which is not Kähler and let $T$ be a

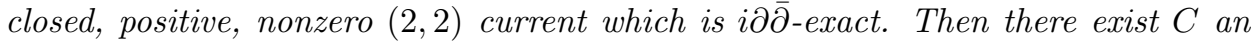
irreducible curve in $X, \lambda>0$ and $R$ a closed positive $(2,2)$ current on $X$ such that $T=\lambda[C]+R$.

Remark 3.1. The above theorem is no longer true in higher dimensions. For instance, let $Y$ be the 3-fold constructed by Hironaka [Hi] which is a proper modification of the projective space $\mathbb{P}^{3}$ and which contains a positive linear combination of curves which is homologuous to 0 . Denote this obstruction by $C$. Let $S$ be an arbitrary Riemann surface and $\omega_{S}$ a positive $(1,1)$ form on $S$. Let $X=Y \times S$ and let $p_{1}$ and $p_{2}$ the two projections. Set $T=p_{1}^{*} C \wedge p_{2}^{*} \omega_{S}$. Then $T$ is a closed positive $(3,3)$ current, which is $i \partial \bar{\partial}$-exact, and it is a residual current.

Remark 3.2. Theorem 3.1 states that on 3-folds, any closed obstruction contains a curve. The above example shows that in higher dimensions there are obstructions which do not contain any curves. It is not clear whether on any manifold in class $\mathcal{C}$ there are obstructions which contain curves. 
Before we prove Theorem 3.1, we need the following:

Proposition 3.2. Let $X$ be a compact complex manifold of dimension $n$ in the Fujiki class $\mathcal{C}$, let $\pi: \widetilde{X} \rightarrow X$ be the blow-up of $X$ along a smooth submanifold $Y$ of dimension $\leq n-2$ and let $\widetilde{Y}$ the exceptional divisor. Let $T$ be a closed positive $(n-1, n-1)$ current on $X$ such that $\chi_{Y} T=0$. Then there exists $\widetilde{T}$ a closed positive $(n-1, n-1)$ current on $\widetilde{X}$ such that $\chi_{\widetilde{Y}} \widetilde{T}=0$ and $\pi_{*} \widetilde{T}=T$. Moreover, $\{\widetilde{T}\}=\pi^{*}\{T\}-\lambda\{[F]\}$, where $F$ is a curve in the fibre of $\pi \mid \widetilde{Y}: \widetilde{Y} \rightarrow Y$ and $\lambda \geq 0$.

Proof. The existence of $\widetilde{T}$ (the strict transform of $T$ ) is proved in AlBa2. Let $F$ be a curve in a fibre of $\pi \mid \widetilde{Y} \rightarrow Y$. We prove that there exists $\lambda \in \mathbb{R}$ such that $\{\widetilde{T}\}=\pi^{*}\{T\}-\lambda\{[F]\}$ by duality. Let $\{\widetilde{\alpha}\} \in H^{1,1}(\widetilde{X})$; it is well-known that $\{\widetilde{\alpha}\}=\pi^{*}\{\alpha\}+\gamma\{[\widetilde{Y}]\}$, where $\{\alpha\} \in H^{1,1}(X)$. Then

$$
\left\langle\{\widetilde{T}\}-\pi^{*}\{T\}-\lambda\{[F]\},\{\widetilde{\alpha}\}\right\rangle=\gamma(\langle\{\widetilde{T}\},\{[\tilde{Y}]\}\rangle+\lambda\langle\{[F]\},\{[\tilde{Y}]\}\rangle) .
$$

It follows that for

$$
\lambda=-\frac{\langle\{\widetilde{T}\},\{[\tilde{Y}]\}\rangle}{\langle\{[F]\},\{[\widetilde{Y}]\}\rangle}
$$

we have $\{\widetilde{T}\}=\pi^{*}\{T\}+\lambda\{[F]\}$. Since $\chi_{\widetilde{Y}} \widetilde{T}=0$, it follows (see AlBa1]) that $\langle\{\widetilde{T}\},\{[\widetilde{Y}]\}\rangle \leq 0$. Now $\langle\{[F]\},\{[\tilde{Y}]\}\rangle<0$ and therefore $\lambda \geq 0$.

Proof of Theorem 3.1. By Siu's decomposition theorem, $T$ can be written $T=$ $\sum_{j} \lambda_{j}\left[C_{j}\right]+R$, where $C_{j}$ are curves in $X, \lambda_{j}>0$ and $R$ is a residual current; i.e., the Lelong sublevel sets $E_{c}(R)$ are 0 dimensional for every $c>0$. So it is enough to prove that $T$ cannot be residual. Suppose $T$ is residual. Then, if $Y$ is a submanifold of $X$ of dimension $\leq 1$, then $\chi_{Y} T=0$. Now suppose that $X$ can be made Kähler by a sequence of blow-ups with smooth centers $X=X_{0} \leftarrow X_{1} \leftarrow \ldots \leftarrow X_{r}$ where $X_{r}$ is Kähler. Denote by $Y_{s}$ the center of the blow-up $X_{s+1} \rightarrow X_{s}$. The centers of the blow-ups are either smooth curves or points. Start off with the current $T=T_{0}$ on $X=X_{0}$. Clearly $\chi_{Y_{0}} T=0$. We construct by induction $i \partial \bar{\partial}$-exact, positive currents $T_{s}$ on $X_{s}$ such that $\chi_{Y_{s}} T_{s}=0$ and $\left(\pi_{s}\right)_{*} T_{s}=T_{s-1}$. Suppose $T_{s}$ has been constructed. From Proposition 3.2 we obtain a closed positive current $T_{s+1}^{\prime}$ on $X_{s+1}$ and $\lambda_{s+1} \geq 0$ such that $T_{s+1}^{\prime}+\lambda_{s+1}\left[F_{s+1}\right]$ is $i \partial \bar{\partial}$-exact and its push-forward is $T_{s}$. If $Y_{s+1} \neq F_{s+1}$, we set $T_{s+1}=T_{s+1}^{\prime}+\lambda_{s+1}\left[F_{s+1}\right]$. If $Y_{s+1}=F_{s+1}$, we set $T_{s+1}=T_{s+1}^{\prime}+\lambda_{s+1}\left[F_{s+1}^{\prime}\right]$, where $F_{s+1}^{\prime} \neq F_{s+1}$ is another curve in the cohomology class $\left\{\left[F_{s+1}\right]\right\}$. It is clear that $\chi_{Y_{s+1}} T_{s+1}=0$ and that $\left(\pi_{s+1}\right)_{*} T_{s+1}=T_{s}$. On $X_{r}$ we obtain a closed positive current $T_{r}$ which is $i \partial \bar{\partial}$-exact. Since $X_{r}$ is Kähler, it follows that $T_{r}=0$ and therefore $T_{0}=T=0$. Contradiction.

\section{REFERENCES}

[AlBa1] Lucia Alessandrini and Giovanni Bassanelli, Compact complex threefolds which are Kähler outside a smooth rational curve, Math. Nachr. 207 (1999), 21-59. MR.1724291 (2001h:32026)

[AlBa2] Lucia Alessandrini and Giovanni Bassanelli, Transforms of currents by modifications and 1-convex manifolds, Osaka J. Math. 40 (2003), no. 3, 717-740. MR2003745|(2004f:32046)

[DePă] Jean-Pierre Demailly and Mihai Paun, Numerical characterization of the Kähler cone of a compact Kähler manifold, Ann. of Math. (2) 159 (2004), no. 3, 1247-1274, DOI 10.4007/annals.2004.159.1247. MR2113021(2005i:32020)

[FiTo] Anna Fino and Adriano Tomassini, A survey on strong KT structures, Bull. Math. Soc. Sci. Math. Roumanie (N.S.) 52(100) (2009), no. 2, 99-116. MR2521810 (2010h:53108) 
[HaLa] Reese Harvey and H. Blaine Lawson Jr., An intrinsic characterization of Kähler manifolds, Invent. Math. 74 (1983), no. 2, 169-198, DOI 10.1007/BF01394312. MR723213 (85b:32013)

[Fu] Akira Fujiki, On automorphism groups of compact Kähler manifolds, Invent. Math. 44 (1978), no. 3, 225-258. MR0481142 (58 \#1285)

[Hi] Heisuke Hironaka, An example of a non-Kählerian complex-analytic deformation of Kählerian complex structures, Ann. of Math. (2) 75 (1962), 190-208. MR0139182 (25 \#2618)

[La] Ahcène Lamari, Courants kählériens et surfaces compactes (French, with English and French summaries), Ann. Inst. Fourier (Grenoble) 49 (1999), no. 1, vii, x, 263-285. MR1688140 (2000d:32034)

[Pe] Thomas Peternell, Algebraicity criteria for compact complex manifolds, Math. Ann. 275 (1986), no. 4, 653-672, DOI 10.1007/BF01459143. MR859336 (88j:32036)

[Va] Jean Varouchas, Kähler spaces and proper open morphisms, Math. Ann. 283 (1989), no. 1, 13-52, DOI 10.1007/BF01457500. MR973802(89m:32021)

Institute of Mathematics of the Romanian Academy, P.O. Box 1-764, Bucharest 014700, ROMANIA

E-mail address: Ionut.Chiose@imar.ro 